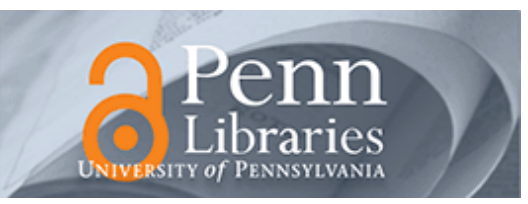

University of Pennsylvania

ScholarlyCommons

April 2007

\title{
Bio-Inspired Group Behaviors for the Deployment of a Swarm of Robots to Multiple Destinations
}

\author{
Spring Berman \\ University of Pennsylvania \\ Ádám Halász \\ University of Pennsylvania \\ R. Vijay Kumar \\ University of Pennsylvania, kumar@grasp.upenn.edu \\ Stephen Pratt \\ University of Pennsylvania
}

Follow this and additional works at: https://repository.upenn.edu/meam_papers

\section{Recommended Citation}

Berman, Spring; Halász, Ádám; Kumar, R. Vijay; and Pratt, Stephen, "Bio-Inspired Group Behaviors for the Deployment of a Swarm of Robots to Multiple Destinations" (2007). Departmental Papers (MEAM). 134. https://repository.upenn.edu/meam_papers/134

Copyright YEAR IEEE. Reprinted from Proceedings of the IEEE International Conference on Robotics and Automation, ICRA '07, April 2007, pages 2318-2323.

This material is posted here with permission of the IEEE. Such permission of the IEEE does not in any way imply IEEE endorsement of any of the University of Pennsylvania's products or services. Internal or personal use of this material is permitted. However, permission to reprint/republish this material for advertising or promotional purposes or for creating new collective works for resale or redistribution must be obtained from the IEEE by writing to pubs-permissions@ieee.org. By choosing to view this document, you agree to all provisions of the copyright laws protecting it.

This paper is posted at ScholarlyCommons. https://repository.upenn.edu/meam_papers/134

For more information, please contact repository@pobox.upenn.edu. 


\title{
Bio-Inspired Group Behaviors for the Deployment of a Swarm of Robots to Multiple Destinations
}

\author{
Abstract \\ We present a methodology for characterizing and synthesizing swarm behaviors using both a \\ macroscopic model that represents a swarm as a continuum and a microscopic model that represents \\ individual robots. We develop a systematic approach for synthesizing behaviors at the macroscopic level \\ that can be realized on individual robots at the microscopic level. Our methodology is inspired by a \\ dynamical model of ant house hunting [1], a decentralized process in which a colony attempts to emigrate \\ to the best site among several alternatives. The model is hybrid because the colony switches between \\ different sets of behaviors, or modes, during this process. At the macroscopic level, we are able to \\ synthesize controllers that result in the deployment of a robotic swarm in a predefined ratio between \\ distinct sites. We then derive hybrid controllers for individual robots using only local interactions and no \\ communication that respect the specifications of the global continuous behavior. Our simulations \\ demonstrate that our synthesis procedure yields a correct microscopic model from the macroscopic \\ description with guarantees on performance at both levels.

\section{Comments} \\ Copyright YEAR IEEE. Reprinted from Proceedings of the IEEE International Conference on Robotics and \\ Automation, ICRA '07, April 2007, pages 2318-2323. \\ This material is posted here with permission of the IEEE. Such permission of the IEEE does not in any way \\ imply IEEE endorsement of any of the University of Pennsylvania's products or services. Internal or \\ personal use of this material is permitted. However, permission to reprint/republish this material for \\ advertising or promotional purposes or for creating new collective works for resale or redistribution must \\ be obtained from the IEEE by writing to pubs-permissions@ieee.org. By choosing to view this document, \\ you agree to all provisions of the copyright laws protecting it.
}




\title{
Bio-Inspired Group Behaviors for the Deployment of a Swarm of Robots to Multiple Destinations
}

\author{
Spring Berman, Ádám Halász, Vijay Kumar, and Stephen Pratt
}

\begin{abstract}
We present a methodology for characterizing and synthesizing swarm behaviors using both a macroscopic model that represents a swarm as a continuum and a microscopic model that represents individual robots. We develop a systematic approach for synthesizing behaviors at the macroscopic level that can be realized on individual robots at the microscopic level. Our methodology is inspired by a dynamical model of ant house hunting [1], a decentralized process in which a colony attempts to emigrate to the best site among several alternatives. The model is hybrid because the colony switches between different sets of behaviors, or modes, during this process. At the macroscopic level, we are able to synthesize controllers that result in the deployment of a robotic swarm in a predefined ratio between distinct sites. We then derive hybrid controllers for individual robots using only local interactions and no communication that respect the specifications of the global continuous behavior. Our simulations demonstrate that our synthesis procedure yields a correct microscopic model from the macroscopic description with guarantees on performance at both levels.
\end{abstract}

\section{INTRODUCTION}

There are many examples of self-organized processes of natural aggregation that achieve global objectives such as nest construction, foraging, brood sorting, hunting, navigation, and emigration, involving only local interactions between individuals and between individuals and their environment. We address the so-called inverse problem for group behaviors, the design of individual behaviors to achieve a desired macroscopic goal. In this paper, we develop a synthesis methodology for autonomous robot behaviors that similarly rely on local interactions without communication but result in provably correct group behaviors. We apply this methodology to a multi-site deployment task, which has applications to the distribution of robots and mobile sensors for search-and-rescue and surveillance.

We are inspired by studies of the process through which an ant colony selects a new home from several sites and emigrates through quorum-dependent recruitment mechanisms [1]. This "house hunting" phenomenon exhibits several desirable features for our task. Although the ants rely only on physical contact and do not employ any communication, simple individual ant behaviors result in group behaviors that are thought to be optimal for the colony. A quorum sensing mechanism speeds up emigration to a site when it is sufficiently populated, a reflection of many individual

\footnotetext{
V. Kumar, Á. Halász, and S. Berman are with the Department of Mechanical Engineering and Applied Mechanics, University of Pennsylvania, Philadelphia, PA 19104 \{kumar, halasz, spring\} egrasp.upenn.edu

S. Pratt is with the School of Life Sciences, Arizona State University, Tempe, AZ 85287 Stephen. Pratteasu.edu
}

decisions on the site's quality. The resulting group behavior is robust to environmental noise and to changes in numbers of agents. These features suggest that a deployment model with quorum-dependent recruitment will produce an effective distribution of resources more quickly and robustly than other approaches.

This work is related in spirit to the work of [2], which presents a systematic approach to translate group behaviors, modeled as vector fields on a low-dimensional abstract manifold, into agent behaviors in a high-dimensional manifold derived from copies of an agent's state space. As in recent work on modeling and analyzing swarm robotic systems [3] [4] [5], we employ a multi-level representation of swarm activity. The highest level consists of a macrocontinuous model, also called the Rate Equation model [6], characterized by differential equations in which the state variables represent population fractions engaged in different roles or tasks. We distinguish the macro-discrete level, which models a discrete number of agents in each role according to the Stochastic Master Equation [7], as an intermediate level. The lowest level is a microscopic model [6] of agents in a physical setting, incorporating the geometry and dynamics of individual agents and possibly modeling heterogeneity. Applications such as collaborative manipulation [3], object clustering [4], and adaptive multi-foraging [5] can be modeled at the macro-continuous level by averaging performances of multiple individual robot controllers at the microscopic level. In contrast to this bottom-up analysis procedure, our methodology is based on a top-down design approach.

In our previous work [11], we employed this three-level representation to synthesize ant-inspired robot behaviors for the task of identifying a single best site. The novel contribution of our work here is a synthesis procedure to alter the group behavior at the macro-continuous level, allowing in turn the synthesis of microscopic level behaviors to achieve the desired group behavior. We apply our methodology to a macro-continuous model of ant house hunting [1]. We alter the house hunting model by adding controls that are designed to meet the requirements of a multi-site deployment task. We realize the specifications of the controlled macro-continuous level behaviors at the macro-discrete level and then at the microscopic level. The resulting agent closed-loop control laws cause the population to behave in the prescribed manner.

\section{METHODOLOGY}

We consider a population of $N$ agents moving in the continuous state space $X_{a} \subset \mathbb{R}^{2}$. At any given time, an agent's actions are determined by one of a set $L_{a}$ of $l_{a}$ controllers or 


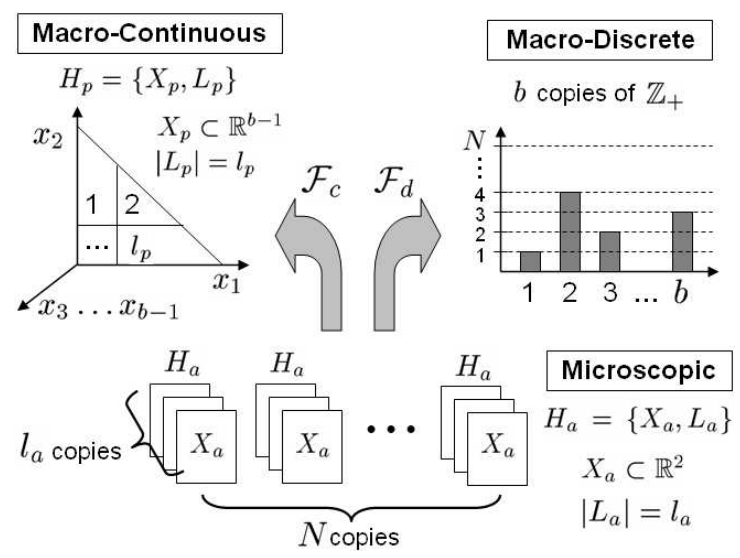

Fig. 1. Levels of abstraction of a swarm

behaviors. We can describe the agent as a hybrid automaton, $H_{a}=\left\{X_{a}, L_{a}\right\}$, to indicate that its activity is governed by both continuous and discrete dynamics. Fig. 1 shows how this high-dimensional microscopic level can be mapped to lower-dimensional representations, the macro-discrete and macro-continuous levels, through the abstractions $\mathcal{F}_{d}$ and $\mathcal{F}_{c}$.

Representing the swarm as a continuous quantity, the macro-continuous level models its dynamics with a set of differential equations whose variables, $x_{i}(i=1, \ldots, b)$, are the population fractions associated with different roles. Each role is defined by a collection of agent controllers. It is assumed that the population is conserved, so one variable may be removed through the conservation constraint. The variables therefore comprise a continuous state space $X_{p} \subset$ $\mathbb{R}^{b-1}$. If the model is a hybrid system, then the state space is divided into a set $L_{p}$ of $l_{p}$ regions, called population modes, each of which is associated with different continuous dynamics. The system may then be described by a hybrid automaton $H_{p}=\left\{X_{p}, L_{p}\right\}$. The macro-discrete level, which considers a swarm as a collection of discrete agents rather than a continuum, maintains a count of the number of agents in each of the $b$ different roles.

\section{Macro-Continuous Level Model}

Our starting point is the model of ant house hunting behavior presented in [1]. This model, constructed from experimental observations of Temnothorax albipennis ants, predicts the behavior of a colony of $N$ ants that is faced with a choice between two new nest sites, labeled 1 and 2, following the destruction of its original nest, site 0 . Site 2 is a higher quality nest than site 1 . A fraction $p$ of the colony is actively involved in house hunting, and the remainder consists of brood items and other "passive" ants that must be carried to a new nest. The active ant fraction is divided among the following state variables: naive ants, $X$; assessors of site $i \in\{1,2\}, Z_{i}$; and recruiters to site $i, Y_{i}$. The passive ant fraction is divided among the variables $B_{i}, i \in\{0,1,2\}$, the passive ants at site $i$.

A salient feature of ant house hunting behavior is the phenomenon of recruitment. Recruiters to site $i$ bring ants from site 0 to $i$, and both their method of recruitment and their target recruitee depend on whether their population has reached a quorum $T$. If $Y_{i}<T$, then the recruiters limit themselves to using tandem runs to lead naive ants, $X$, to assess site $i$. If $Y_{i} \geq T$, the recruiters use the faster method of transports to carry the passive ants at site $0, B_{0}$, to site $i$.

The model equations are as follows [1]:

$$
\begin{aligned}
\dot{X}= & -\left(\mu_{1}+\mu_{2}\right) X-\lambda_{1} Y_{1} \theta(X) \theta\left(T-Y_{1}\right) \\
& -\lambda_{2} Y_{2} \theta(X) \theta\left(T-Y_{2}\right) \\
\dot{Y}_{1}= & k_{1} Z_{1}-\rho_{12} Y_{1} \\
\dot{Y_{2}}= & k_{2} Z_{2}+\rho_{12} Y_{1} \\
\dot{Z}_{1}= & \mu_{1} X+\lambda_{1} Y_{1} \theta(X) \theta\left(T-Y_{1}\right)-\rho_{12} Z_{1}-k_{1} Z_{1} \\
\dot{Z}_{2}= & \mu_{2} X+\lambda_{2} Y_{2} \theta(X) \theta\left(T-Y_{2}\right)+\rho_{12} Z_{1}-k_{2} Z_{2} \\
\dot{B}_{0}= & -\phi_{1} Y_{1} \theta\left(B_{0}\right) \theta\left(Y_{1}-T\right)-\phi_{2} Y_{2} \theta\left(B_{0}\right) \theta\left(Y_{2}-T\right) \\
\dot{B}_{1}= & \phi_{1} Y_{1} \theta\left(B_{0}\right) \theta\left(Y_{1}-T\right) \\
\dot{B}_{2}= & \phi_{2} Y_{2} \theta\left(B_{0}\right) \theta\left(Y_{2}-T\right)
\end{aligned}
$$

In these equations, $\theta_{a}(X)=1$ when $X>0$ and 0 otherwise. Naive ants discover site $i$ at per capita rate $\mu_{i}$. Assessors become recruiters to site $i$ at per capita rate $k_{i}$, which is directly related to the quality of the site. $\lambda_{i}$ and $\phi_{i}$ are the per capita rates at which recruiters perform tandem runs and transports to site $i$, respectively. $\rho_{i j}$ is the per capita rate at which assessors and recruiters of site $i$ encounter site $j$ and switch their allegiance by becoming assessors and recruiters, respectively, of that site.

In a robotics context, the active ants are analogous to robots that organize the distribution of resources or other robots, the "passive" agents, among multiple sites. In the most general case, we may imagine a scenario in which there are $M$ sites and $N>>$ robots located at an initial site 0 . One may specify a connectivity graph that describes how robots can move between sites; this graph need not be complete, but all sites must be connected. The goal is to deploy the robots so that the robot fraction at each site is as close as possible to a predetermined fraction. This balanced deployment paradigm may be used for tasks such as the allocation of resources proportionally to local necessities or the surveillance of several sites of similar importance.

We consider the special case of the house hunting model, in which $M=2$ and both sites are connected to site 0 . Fig. 2 (left) shows a typical environment with sites 0,1 , and 2 and obstacles. We only consider environments that lend themselves to the construction of navigation functions [12] and the abstraction of a circular boundary with circular obstacles (right).

\section{Macro-Continuous LeVel BehaVior Synthesis}

\section{A. Specification for balanced deployment of robots}

We extend the ant house hunting model to allow the swarm to split between two available sites at a predetermined occupancy ratio $\alpha \geq 1$. We focus on the active robots only and require that the system has one equilibrium at:

$$
X=Z_{1}=Z_{2}=0, \quad Y_{1}=\frac{p}{1+\alpha}, \quad Y_{2}=\frac{\alpha p}{1+\alpha},
$$

Due to the switching functions that control the tandem run and transport terms, model (1) is a hybrid system $H_{p}$. 


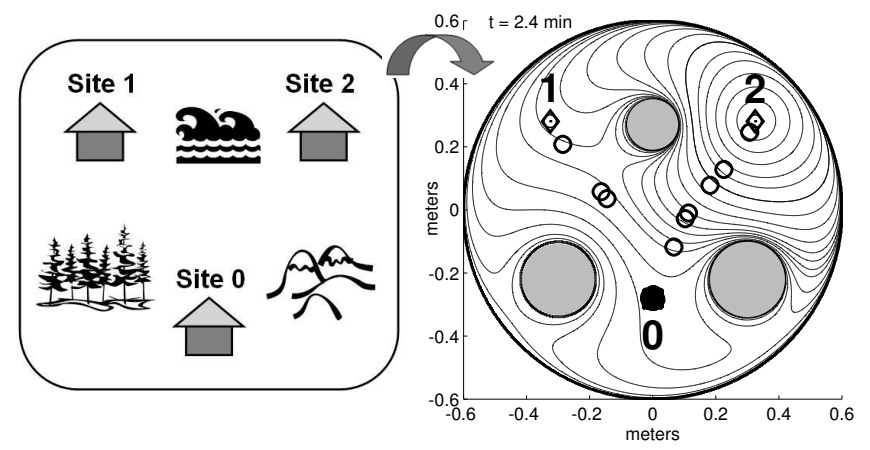

Fig. 2. A three-site environment with obstacles (left) and the model with the contours of a navigation function (right) $[\mathrm{O}=$ naive robot, $\diamond=$ assessor $]$.

The five active robot state variables $\left\{X, Y_{1}, Y_{2}, Z_{1}, Z_{2}\right\}$ are decoupled from the three passive robot state variables $\left\{B_{0}, B_{1}, B_{2}\right\}$. Since the active robot fraction $p$ is constant, $X$ can be eliminated through the conservation constraint:

$$
X+Y_{1}+Y_{2}+Z_{1}+Z_{2}=p .
$$

The system therefore evolves on the four-dimensional state space $\left\{Y_{1}, Y_{2}, Z_{1}, Z_{2} \geq 0, Y_{1}+Y_{2}+Z_{1}+Z_{2} \leq p\right\}$. The state space is divided into four modes by the hyperplanes $Y_{1}=T$ and $Y_{2}=T$, the quorum switches. The modes are labeled by combinations of $Q$ and $N$, where $Q$ stands for "quorum reached" and $N$ stands for "no quorum":

$$
\begin{array}{ll}
N N: Y_{1}<T, Y_{2}<T & Q N: Y_{1} \geq T, Y_{2}<T \\
N Q: Y_{1}<T, Y_{2} \geq T & Q Q: Y_{1} \geq T, Y_{2} \geq T .
\end{array}
$$

We require that the equilibrium (2) is inside mode $Q Q$; that is, $(1+\alpha) T \leq p \leq \alpha p$.

\section{B. Synthesis of new behaviors}

We first add controls to the dynamics of mode $Q Q$ so that it contains steady state (2) and no trajectories leave the mode. Mode $Q Q$ consists of an affine dynamical system $\dot{x}=$ $A x+a$ evolving on a four-dimensional simplex. Here $x=$ $\left[\begin{array}{lllll}Y_{1} & Y_{2} & Z_{1} & Z_{2}\end{array}\right]^{T}$, and the offset $a$ is obtained by replacing $X$ with $p-Y_{1}-Y_{2}-Z_{1}-Z_{2}$ in model (1), according to equation (3). We redefine the dynamics of $Q Q$ as the control system $\dot{x}=A x+B u+a$, where $u=F x+g$ is an affine feedback law. The controlled dynamics are thus:

$$
\dot{x}=(A+B F) x+(a+B g) .
$$

To enforce the conservation law (3), we dictate that the controls must result in a balance of terms among the differential equations. We only add controls to the recruiter dynamics, since the recruiter fractions alone determine the current mode and the steady state:

$$
\begin{aligned}
& \dot{Y}_{1}=k_{1} Z_{1}-\rho_{12 c} Y_{1}+\rho_{21 c} Y_{2}+d \\
& \dot{Y}_{2}=k_{2} Z_{2}+\rho_{12 c} Y_{1}-\rho_{21 c} Y_{2}-d
\end{aligned}
$$

$Y_{2}$ robots can now switch allegiance to $Y_{1}$ at rate $\rho_{21 c}$. $\rho_{12 c}$ may differ from $\rho_{12}$ in the original model, and $d$ is a constant.
To ensure the desired steady state, we set $\dot{Y}_{1}=0, \dot{Y}_{2}=0$ and substitute the values in equation (2) for the variables. This results in the equation:

$$
\left(\alpha \rho_{21 c}-\rho_{12 c}\right) \frac{p}{1+\alpha}+d=0 .
$$

To prevent trajectories from escaping mode $Q Q$, we apply conditions (1b) and (2a) of Proposition 3.1 from [9] at the facets $F_{1}=\left\{x \in \mathbb{R}^{4} \mid n_{1}^{T} x=-T\right\}$ and $F_{2}=$ $\left\{x \in \mathbb{R}^{4} \mid n_{2}^{T} x=-T\right\}$, where $n_{1}=\left[\begin{array}{cccc}-1 & 0 & 0 & 0\end{array}\right]^{T}$ and $n_{2}=\left[\begin{array}{llll}0 & -1 & 0 & 0\end{array}\right]^{T}$ are the normal vectors of $F_{1}$ and $F_{2}$, respectively. The conditions reflect the fact that for a point $x$ of a simplex, an affine function $f(x)$ is a convex combination of the values of $f$ at the simplex vertices. The vertices of the simplex corresponding to mode $Q Q$ are:

$$
\begin{aligned}
& v_{1}=\left[\begin{array}{llll}
T & T & 0 & 0
\end{array}\right]^{T} \\
& v_{4}=\left[\begin{array}{llll}
T(p N-T) & 0 & 0
\end{array}\right]^{T} \\
& v_{2}=[T T(p N-2 T) 0]^{T} \quad v_{5}=[(p N-T) T 00]^{T} \\
& v_{3}=\left[\begin{array}{lll}
T & 0 & (p N-2 T)
\end{array}\right]^{T}
\end{aligned}
$$

Setting $f(x)=\dot{x}$ from equation (4) and noting that $\dot{x}=$ $\left[\begin{array}{llll}\dot{Y}_{1} & \dot{Y}_{2} & \dot{Z}_{1} & \dot{Z}_{2}\end{array}\right]^{T}$, the conditions to be satisfied are:

$$
\begin{gathered}
n_{1}^{T} f\left(v_{i}\right) \leq 0, \quad i \in\{1,2,3,4\} \quad \Rightarrow \quad \dot{Y}_{1} \geq 0 \\
n_{2}^{T} f\left(v_{j}\right) \leq 0, \quad j \in\{1,2,3,5\} \quad \Rightarrow \quad \dot{Y}_{2} \geq 0,
\end{gathered}
$$

where $\dot{Y}_{1}$ and $\dot{Y}_{2}$ are evaluated at the designated vertices using the equations in (5). The resulting set of inequalities is satisfied if conditions (8) and (9) are satisfied when evaluated only at vertex $v_{1}$ :

$$
\begin{aligned}
\dot{Y}_{1} & =-\rho_{12 c} T+\rho_{21 c} T+d \geq 0 \\
\dot{Y}_{2} & =\rho_{12 c} T-\rho_{21 c} T-d \geq 0 \\
& \Rightarrow d=\left(\rho_{12 c}-\rho_{21 c}\right) T .
\end{aligned}
$$

The relationship between $\rho_{12 c}$ and $\rho_{21 c}$ may be derived by substituting the expression for $d$ from equation (12) into equation (6):

$$
\frac{\rho_{12 c}}{\rho_{21 c}}=\frac{\alpha p-T(1+\alpha)}{p-T(1+\alpha)} .
$$

We now modify the dynamics of the other three modes so that they contain no attractors and trajectories starting inside these modes follow the pattern: $N N \rightarrow N Q$ or $Q N$, $Q N \rightarrow Q Q, N Q \rightarrow Q Q$. We do this by replacing the recruiter dynamics in these modes with the equations in (5) with switches that prevent states from flowing in the $-Y_{1}$ direction in modes $N N, N Q$ and in the $-Y_{2}$ direction in modes $N N, Q N$.

The controlled system is thus defined by the equations:

$$
\dot{X}, \dot{Z}_{1}, \dot{Z}_{2} \text { from model (1) }
$$

$\dot{Y}_{1}=k_{1} Z_{1}-\rho_{12 c} Y_{1} \theta\left(Y_{1}-T\right)+\left(\rho_{21 c} Y_{2}+d\right) \theta\left(Y_{2}-T\right)$

$\dot{Y}_{2}=k_{2} Z_{2}+\rho_{12 c} Y_{1} \theta\left(Y_{1}-T\right)-\left(\rho_{21 c} Y_{2}+d\right) \theta\left(Y_{2}-T\right)$

We replace $\rho_{12}$ in the $\dot{Z}_{1}, \dot{Z}_{2}$ equations with $\rho_{12 c}$.

Fig. 3 displays trajectories of the systems (1) and (14) on a 2-D projection of the state space. The thick solid line is 


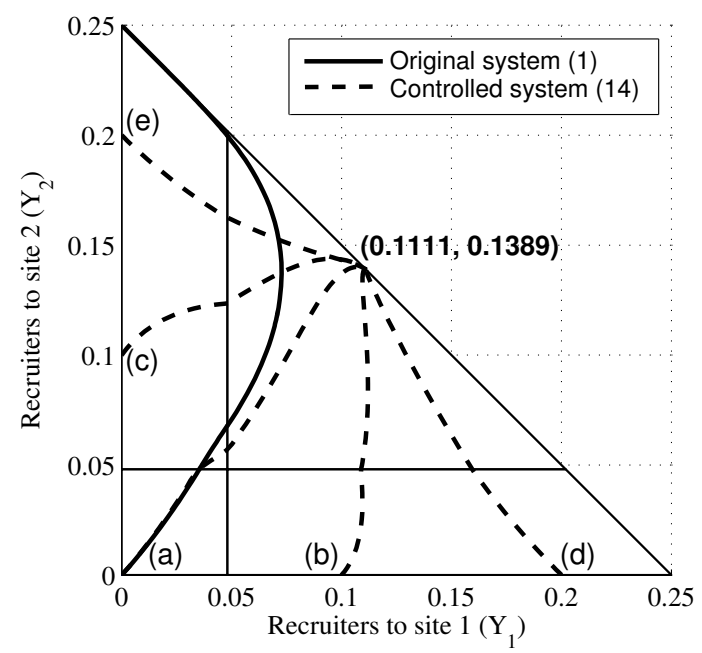

Fig. 3. Trajectories of the original and modified macro-continuous models with $p=0.25, T=0.0481, \mu_{1}=\mu_{2}=0.013, \lambda_{1}=\lambda_{2}=0.033, k_{1}=$ $0.019, k_{2}=0.020$ (values are from [1] [10]); $\alpha=1.25, \rho_{12 c}=0.01$, $\rho_{21 c}=0.0069, d=0.000147$. The dashed lines are trajectories beginning at (a) $\left[\begin{array}{llll}0 & 0 & 0 & 0\end{array}\right]^{T}$, (b) $\left[\begin{array}{llll}0.1 & 0 & 0.1 & 0\end{array}\right]^{T}$, (c) $\left[\begin{array}{llll}0 & 0.1 & 0 & 0.1\end{array}\right]^{T}$, (d) $\left[\begin{array}{llll}0.2 & 0 & 0 & 0\end{array}\right]^{T}$, and (e) $\left[\begin{array}{llll}0 & 0.2 & 0 & 0\end{array}\right]^{T}$.

the trajectory of system (1) beginning at $x=\left[\begin{array}{llll}0 & 0 & 0 & 0\end{array}\right]^{T}$; all other initial conditions produce the same steady state. The dashed lines are sample trajectories of system (14) for $p=0.25, \alpha=1.25$. The figure shows that the original system converges to the equilibrium $\left[\begin{array}{lllll}0 & 0.25 & 0 & 0\end{array}\right]^{T}$, whereas the controlled system converges to the equilibrium (2).

Time delays corresponding to the travel time between sites were ignored in our synthesis procedure. However, they were included in the macro-continuous simulations in Section VI by modifying the controlled model to be a set of delay differential equations in which the variables represent robots that are physically at a site. Time delays do not influence the location of steady states, but they may induce limit cycles, although none arise in this model. The effect of time delays on behavior synthesis will be addressed in future work.

\section{DESIGN OF Individual Robot Behaviors}

The following sections describe how to translate the macro-continuous model into a macro-discrete model that accounts for an integer number of robots and then into a microscopic model of individual robots.

\section{A. Macro-Discrete Level}

Gillespie's Direct Method [7] was used to perform a stochastic simulation of the system that is represented deterministically by the macro-continuous controlled model. The method replaces the continuous rate equations with a sequence of individual robot state transitions and their times. In our model, transition times for switching roles are governed by a Poisson distribution. As $N \rightarrow \infty$, the Poisson transition probabilities per unit time become transition rates, and the macro-discrete level predictions approach the macrocontinuous level solution.

Each transition $s$ between roles is associated with a propensity $a_{s}$, which is is defined such that $a_{s} d t$ is the probability that transition $s$ will occur in the next time interval $d t$. We describe how to compute the propensities in [11]. The next state transition is selected according to a uniform probability distribution over the propensities, and the time until its occurrence, $\Delta \tau$, is computed from an exponential distribution with $\sum_{s} a_{s}$ as its parameter. The time is advanced by $\Delta \tau$ and the transition between two roles is simulated by decrementing the number of robots in one role and incrementing the number in the second. Depending on the transition, the increment may occur immediately or at a deterministic time in the future that represents the completion of the robot's navigation between sites. A transition associated with a tandem run or transport is initiated independently of the current availability of recruitees at site 0 , since a recruiter at a new site would not know this information. Such a transition causes a decrement in the recruitee population only if a recruitee is available at the time when the recruiter is expected to arrive at site 0 .

\section{B. Modeling Individual Robots}

A robot is represented as an entity that stores knowledge of its role, site 0 , another site, position, speed, and whether it is navigating to a site. The stochastic simulation method described in Section V-A is used to generate state transitions and their times. The simulation is run in time steps $\Delta t$ to implement the robots' incremental navigation through their environment, so a transition at time $\tau$ is initiated when $t \leq$ $\tau<t+\Delta t$. Each transition is assigned to a random robot in the appropriate role that is not already en route to a site.

Navigation functions [12] are used to generate robot trajectories between sites. A navigation function provides a form for a feedback controller that guides an agent to a goal, the unique minimum of the function, while preventing collisions with obstacles. The position $r$ of a robot, which is represented as a point, is updated at each time step by numerically integrating the equation

$$
\dot{r}=-v \nabla \varphi_{\kappa}\left(r, r_{d}\right) /\left\|\nabla \varphi_{\kappa}\left(r, r_{d}\right)\right\|,
$$

where $v$ is the robot's speed and $\varphi_{\kappa}\left(r, r_{d}\right)$ is the navigation function with the ant's current point destination $r_{d}$. The parameter $\kappa$, which was selected empirically, must be high enough to make $\varphi$ a valid navigation function and to eliminate local minima. Various combinations of $v$ and $r_{d}$ are used to produce different agent controllers; for example, one $l \in L_{a}$ would be navigating from site 0 to site 1 at the tandem-running speed.

Comment: Although our microscopic level simulation uses a "global planner" to initiate transitions, it has an equivalent formulation that can be implemented on a decentralized swarm. The advantage of the Gillespie method is its speed; unlike the decentralized approach, it does not require looping through all agents at each time step. We also note that although our model still requires more detail, including quorum estimation, recruiter-recruitee communication, and interrobot collision avoidance, the quorum dependency does not pose a theoretical impediment to synthesizing an executable robot controller. See [11] for more details on these topics. 


\section{RESULTS}

We implemented macro-continuous, macro-discrete, and microscopic level simulations in Matlab of the controlled model (14) for an occupancy ratio $\alpha=1.25$. We used the three-site, three-obstacle environment shown on the right side of Fig. 2; the robots are modeled at the scale of ants. The sites are $65 \mathrm{~cm}$ apart, the inter-site distance used in experiments to derive the site discovery and recruitment rates [10]. Each site is represented as a circle of radius $0.02 \mathrm{~m}$; a robot is considered inside the site once it enters the circle.

All robots begin as naive at site 0 . Robots performing tandem runs move at $1.5 \mathrm{~mm} / \mathrm{sec}$, while all other robots move at $4.6 \mathrm{~mm} / \mathrm{sec}$, the transport speed [1]. The rate units are $\mathrm{min}^{-1}$. In the macro-continuous and macro-discrete simulations, the time delays $\tau_{i j}$ due to navigation from site $i$ to site $j$ were measured from the microscopic level simulation. The delays due to tandem runs are $\tau_{01}=\tau_{02}=6$ min; delays from all other journeys are $\tau_{01}=\tau_{02}=2.2 \mathrm{~min}$, $\tau_{10}=\tau_{20}=2.5 \mathrm{~min}$, and $\tau_{12}=\tau_{21}=2.48 \mathrm{~min}$. The time step $\Delta t$ is $0.05 \mathrm{~min}$.

Fig. 4 displays the recruiter fractions at sites 1 and 2 from simulations at all three levels with an active robot population of $p N=208$. The macro-discrete and microscopic level simulations match the macro-continuous model fairly well. Although not shown, it has been verified that the macrodiscrete simulation approaches the macro-continuous model as $p N$ increases. At $700 \mathrm{~min}$ in the macro-continuous model, $Y_{1}=0.1089$ and $Y_{2}=0.1357$, which fall short of the steady-state values in (2) by the fraction of recruiters that are traveling between sites 1 and 2 . These robots may be thought of as patrolling the inter-site area. The final values of $Y_{1}$ and $Y_{2}$ in the other two simulations are close to these fractions. Thus, the steady-state occupancy ratio in the macro-discrete and microscopic models approximates $\alpha=1.25$.

To investigate the effect of robot population on the steadystate recruiter fractions, macro-discrete simulations were run for active robot populations of 52, 208, and 832. For each run, $Y_{1}$ and $Y_{2}$ were sampled at intervals as close as possible to 2 min from 1000 to $5000 \mathrm{~min}$. Fig. 5 shows the resulting frequency distributions of $Y_{1}$ and $Y_{2}$. The vertical lines near the centers of the distributions mark the macro-continuous steady-state values. The mean and standard deviation of each variable are also recorded in the figure. The standard deviations decrease as the number of robots increases, which is consistent with the fact that the macro-continuous model is obtained as the limit of the macro-discrete model. The standard deviation is less than $15 \%$ of the mean for a relatively modest population of 52 active robots and less than $4 \%$ for a larger but still realistic population of 832 .

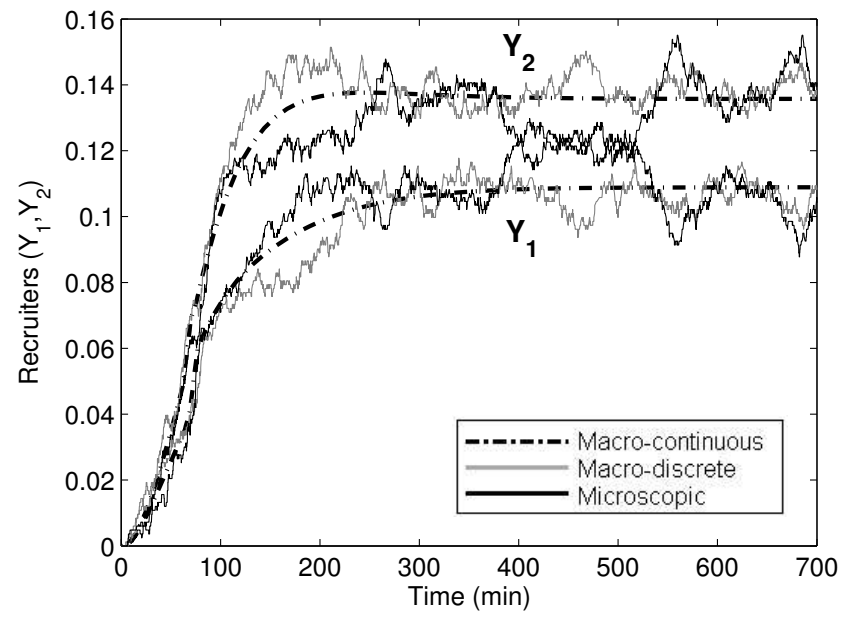

Fig. 4. Recruiter fractions $Y_{1}, Y_{2}$; parameters are the same as in Fig. 3.
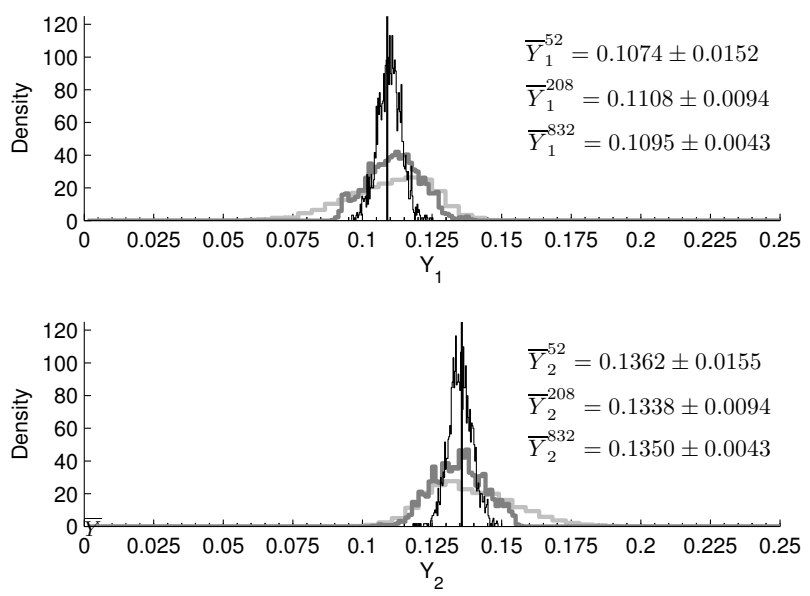

Fig. 5. Histograms of steady-state $Y_{1}$ and $Y_{2}$ for $p N=52$ (light gray), 208 (dark gray), and 832 (black), where $p=0.25$. Bar width is $1 / N$ for each value of $N$. Vertical lines mark $Y_{1}=0.1089$ and $Y_{2}=0.1357$.

\section{DISCUSSION}

We have described abstractions of a robotic swarm at three levels and presented a methodology for synthesizing individual robot behaviors. Our main contribution is the top-down approach to designing robot behaviors. We have illustrated this methodology with the control and simulation of site population growth in a model of ant house hunting adapted to a robotic setting where robots must be deployed to multiple sites in a specified ratio.

An interesting question is whether the features of ant house hunting actually provide an advantage in fulfilling the task of balanced deployment. For example, consider the simpler 


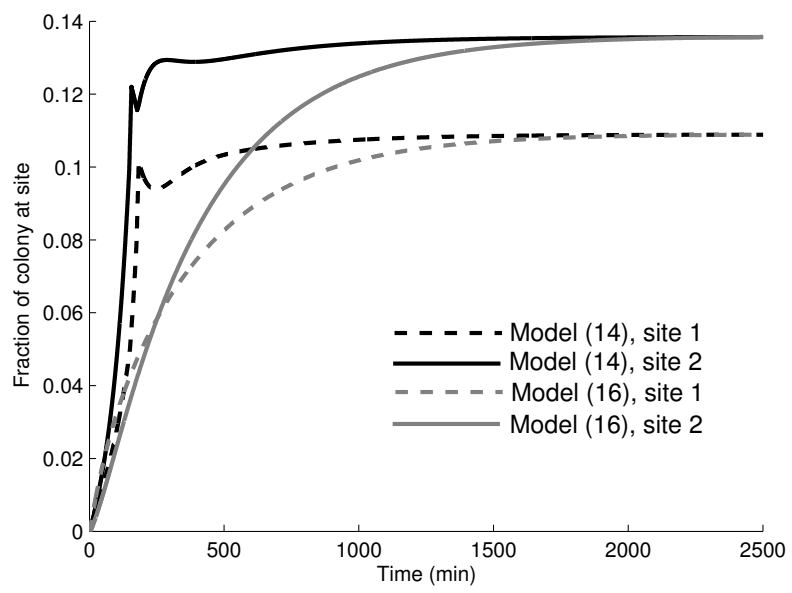

Fig. 6. Population fractions at sites 1 and 2; parameters are the same as in Fig. 3 except for $\mu_{1}=\mu_{2}=0.0013$.

linear feedback law, which does not rely on quorum sensing or switching between controllers but has the desired steady state fractions of $X, Y_{1}$, and $Y_{2}$ from (2):

$$
\begin{aligned}
\dot{X} & =-\left(\mu_{1}+\mu_{2}\right) X \\
\dot{Y}_{1} & =\mu_{1} X-\rho_{12 c} Y_{1}+\rho_{21 c} Y_{2}+d \\
\dot{Y_{2}} & =\mu_{2} X+\rho_{12 c} Y_{1}-\rho_{21 c} Y_{2}-d
\end{aligned}
$$

where $\rho_{12 c}, \rho_{21 c}$ and $d$ satisfy equation (6). It would be possible to use (16) as our macro-continuous model and synthesize robot behaviors from it using our methodology. Indeed, for the particular set of parameters used in our simulations, the difference between models (14) and (16) is not substantial. However, if the discovery rates $\mu_{1}$ and $\mu_{2}$ in both models are reduced by a factor of 10 , the importance of tandem runs becomes apparent. Fig. 6 shows simulation results ${ }^{1}$ of $\left(Z_{i}+Y_{i}\right)(i=1,2)$ for model (14) and $Y_{i}(i=1,2)$ for model (16) in this situation. The quorum-based model presented here converges significantly faster to the desired steady state. This illustrates a possible advantage of the strategy employed by ants over the more obvious linear feedback law (16). The recruitment rates, which are controllable parameters, can significantly speed up the deployment process to compensate for a low discovery rate arising from environmental constraints. The recruitment paradigm can be shown to increase robustness to perturbations in the environment and robot population and to noise in sensing and control of individual agents.

\footnotetext{
${ }^{1}$ Both models were simulated as delay differential equations with the previously defined time delays to include the effect of navigation.
}

In future work, we would like to extend our synthesis methodology to systems for which the macro-discrete level is characterized by multi-modal distributions, which may not be well-represented by macro-continuous abstractions [7]. This is almost always the case in relatively small populations, in which the dynamics are best described by stochastic transitions between multiple stable steady states [8]. Finally, we note that the methodology in this paper extends to higherdimensional state spaces at the macro-continuous level [9], such as the more detailed house hunting model in [11].

Acknowledgements: We gratefully acknowledge the support of NSF grants CCR02-05336 and IIS-0427313, and ARO Grants W911NF-05-1-0219 and W911NF-04-1-0148.

\section{REFERENCES}

[1] Franks, N., Pratt, S., Mallon, E., Britton, N., Sumpter, D.: Information flow, opinion polling and collective intelligence in house-hunting social insects. Phil Trans Roy Soc London B 357 (2002) 1567-1584

[2] Belta, C., Kumar, V.: Abstraction and control for groups of robots. IEEE Transactions on Robotics 20:5 (2004) 865-875

[3] Martinoli, A., Easton, K., Agassounon, W.: Modeling of swarm robotic systems: a case study in collaborative distributed manipulation. Special issue on Experimental Robotics, Int. Journal of Robotics Research 23(4) (2004). B. Siciliano (ed.) 415-436

[4] Agassounon, W., Martinoli, A., Easton, K.: Macroscopic modeling of aggregation experiments using embodied agents in teams of constant and time-varying sizes. Autonomous Robots 17(2-3) (2004). M. Dorigo, E. Sahin (eds.) 163-191

[5] Lerman, K., Jones, C., Galstyan, A., Mataric, M.: Analysis of dynamic task allocation in multi-robot systems. Int. J. of Robotics Research, 25(4) (2006) 225-242

[6] Lerman, K., Martinoli, A., Galstyan, A.: A review of probabilistic macroscopic models for swarm robotic systems. In Swarm Robotics Workshop: State-of-the-art Survey, LNCS 3342 (2005). E. Sahin, W. Spears (eds.) 143-152

[7] Gillespie, D.: A general method for numerically simulating the stochastic time evolution of coupled chemical reactions. J Comp Physics 22 (1976) 403-434

[8] Julius, A., Halász, A., Kumar, V., Pappas, G.: Finite state abstraction of a stochastic model of the lactose regulation system of Escherichia coli. IEEE Conf on Dec and Control, San Diego, Dec. 2006. To appear.

[9] Habets, L., and van Schuppen, J.: A control problem for affine dynamical systems on a full-dimensional polytope. Automatica $\mathbf{4 0}$ (2004) 21-35

[10] Pratt, S., Mallon, E., Sumpter, D., Franks, N.: Quorum sensing, recruitment, and collective decision-making during colony emigration by the ant Leptothorax albipennis. Behav Ecol Sociobiol 52 (2002) $117-127$

[11] Berman, S., Halász, A., Kumar, V., Pratt, S.: Algorithms for the analysis and synthesis of a bio-inspired swarm robotic system. Swarm Robotics SAB 2006 Int'l Workshop, Rome, Italy. To appear in LNCS.

[12] Rimon, E., Koditschek, D.: Exact robot navigation using artificial potential functions. IEEE Transactions on Robotics and Automation 8(5) (1992) 501-518

[13] Parrish, J., Hamner, W. (eds.): Animal Groups in Three Dimensions. Cambridge University Press, New York (1997) 